\title{
INITIAL RADIO OBSERVATIONS OF SN1987a IN THE LARGE MAGELLANIC CLOUD
}

\author{
A. J. Turtle and D. Campbell-Wilson \\ School of Physics, Sydney University, Sydney 2006 \\ J. D. Bunton \\ Department of Electrical Engineering, Sydney University, Sydney 2006 \\ D. L. Jauncey, M. J. Kesteven, R. N. Manchester, R. P. Norris, \\ M. C. Storey, and G. L. White \\ Division of Radiophysics, CSIRO, P.O. Box 76, Epping, 2121 \\ J. E. Reynolds \\ M.S.S.S.O., Private Bag, Woden, ACT, 2606 \\ D. F. Malin \\ Anglo-Australian Observatory, P.O. Box 296, Epping, 2121
}

\begin{abstract}
A prompt radio burst has been observed from the supernova 1987a in the Large Magellanic Cloud. Observations were made at $0.843,1.415,2.29$, and $8.41 \mathrm{GHz}$. At frequencies around $1 \mathrm{GHz}$, the peak flux density reached about $150 \mathrm{mJy}$ and occurred within four days of the supernova. This event may be a weak precursor to a major radio outburst of the type previously observed in other extragalactic supernovae. Radio monitoring of the supernova is continuing at each of the above frequencies, and coordination is underway of a southern hemisphere VLBI array to map the radio outburst region as it expands. Differential astrometry carried out on prime-focus plates taken with the Anglo-Australian telescope indicates that the component, star 1, of Sanduleak's star SK-69202 is within $0.05 \pm 0.13$ arcsec of the supernova.
\end{abstract}

A complete discussion of our observations can be found in Nature, 327, 38-40 (1987) and Nature, 327, 36-38 (1987). 